\title{
Efeito da avaliação nutricional sobre o tempo de internação hospitalar: Revisão sistemática
}

\author{
Effect of nutritional assessment on length of hospital stay: Systematic review \\ Efecto de la evaluación nutricional sobre la duración de la estancia hospitalaria:
} Revisión sistemática

Recebido: 24/05/2021 | Revisado: 01/06/2021 | Aceito: 05/06/2021 | Publicado: 20/06/2021

Érica Tamires Gomes de Araújo

ORCID: https://orcid.org/0000-0001-8846-4632 Universidade Federal de Campina Grande, Brasil E-mail: ericatgaraujo@gmail.com

Itiel de Souza Aquino

ORCID: https://orcid.org/0000-0003-3839-3666 Universidade Federal de Campina Grande, Brasil E-mail: itiel.aquino@gmail.com

Randolfo Randall Farias Ferreira Brito ORCID: https://orcid.org/0000-0003-0452-1527 Universidade Federal de Campina Grande, Brasil E-mail: randolfobrito@hotmail.com

Rosane Silva de Oliveira Teixeira ORCID: https://orcid.org/0000-0001-8470-5785 Universidade Federal de Campina Grande, Brasil E-mail: rosaneteixeira7890@ gmail.com

Erik Trovão Diniz

ORCID: https://orcid.org/0000-0003-2147-227X Universidade Federal de Campina Grande, Brasil E-mail: erik_td@hotmail.com

\begin{abstract}
Resumo
A desnutrição em pacientes internados correlaciona-se com o aumento da frequência de intercorrências clínicas e mortalidade, além de estar associada a um maior período de hospitalização, maior risco de infecções e aumento dos custos para o serviço de saúde. O objetivo desta pesquisa foi elaborar revisão sistemática sobre o efeito da avaliação nutricional sobre o tempo de internação hospitalar e caracterizar a prevalência de desnutrição e risco nutricional nos pacientes hospitalizados. Foi realizada uma revisão sistemática de artigos originais utilizando os descritores "Nutrition Assessment", "Length of stay" " "Prognosis", nas bases de dados da Bireme/BVS, publicados em Inglês, Português ou Espanhol. De um total de 2.181 pacientes analisados nos estudos selecionados, cerca de $57 \%$ tinham idade igual ou maior a 60 anos, com média variando entre 63 e 85 anos. A prevalência de desnutrição ou de risco nutricional da população geral estudada variou entre $0,98 \%$ a $69,9 \%$, a depender do instrumento de avaliação nutricional utilizado na análise. Em relação ao tempo de internação hospitalar e sua associação com o grau de desnutrição ou risco nutricional, evidenciou-se correlação positiva na maioria das séries. A elevada prevalência da desnutrição à admissão hospitalar parece acarretar em maior tempo de internação dos pacientes, elevação de custos hospitalares e pior do desfecho clínico. Apesar de não existir um modelo de avaliação nutricional adequado, sua importância é indiscutível e estudos futuros são necessários para elucidar melhor o tema.
\end{abstract}

Palavras-chave: Avaliação nutricional; Tempo de internação; Prognóstico; Desnutrição.

\begin{abstract}
Malnutrition in hospitalized patients correlates with the increased frequency of clinical complications and mortality, in addition to being associated with a longer period of hospitalization, greater risk of infections and increased costs for the health service. The objective of this research was to elaborate a systematic review on the effect of nutritional assessment on the length of hospital stay and to characterize the prevalence of malnutrition and nutritional risk in hospitalized patients. A systematic review of original articles was carried out using the descriptors "Nutrition Assessment", "Length of stay" and "Prognosis", in the Bireme / VHL databases, published in English, Portuguese or Spanish. Of a total of 2,181 patients analyzed in the selected studies, about $57 \%$ were aged 60 years or over, with an average ranging between 63 and 85 years. The prevalence of malnutrition or nutritional risk in the general population studied ranged from $0.98 \%$ to $69.9 \%$, depending on the nutritional assessment instrument used in the analysis. Regarding the length of hospital stay and its association with the degree of malnutrition or nutritional risk, there was a positive correlation in most of the
\end{abstract}


series. The high prevalence of malnutrition at hospital admission seems to lead to longer hospital stays for patients, higher hospital costs and worse clinical outcome. Although there is no adequate nutritional assessment model, its importance is indisputable and further studies are needed to better elucidate the topic.

Keywords: Nutritional Assessment; Hospitalization time; Prognosis; Malnutrition.

\section{Resumen}

La desnutrición en los pacientes hospitalizados se correlaciona con el aumento de la frecuencia de complicaciones clínicas y la mortalidad, además de estar asociada a un mayor período de hospitalización, mayor riesgo de infecciones y mayores costos para el servicio de salud. El objetivo de esta investigación fue elaborar una revisión sistemática sobre el efecto de la evaluación nutricional en la duración de la estancia hospitalaria y caracterizar la prevalencia de desnutrición y riesgo nutricional en pacientes hospitalizados. Se realizó una revisión sistemática de artículos originales utilizando los descriptores "Nutrition Assessment", "Length of stay" y "Prognosis", en las bases de datos Bireme / VHL, publicados en inglés, portugués o español. De un total de 2.181 pacientes analizados en los estudios seleccionados, alrededor del $57 \%$ tenían 60 años o más, con un promedio que oscilaba entre 63 y 85 años. La prevalencia de desnutrición o riesgo nutricional en la población general estudiada osciló entre 0,98\% y 69,9\%, según el instrumento de valoración nutricional utilizado en el análisis. En cuanto a la duración de la estancia hospitalaria y su asociación con el grado de desnutrición o riesgo nutricional, hubo correlación positiva en la mayoría de las series. La alta prevalencia de desnutrición al ingreso hospitalario parece conducir a estadías hospitalarias más prolongadas de los pacientes, mayores costos hospitalarios y peores resultados clínicos. Aunque no existe un modelo de valoración nutricional adecuado, su importancia es indiscutible y se necesitan más estudios para dilucidar mejor el tema.

Palabras clave: Evaluación nutricional; Tiempo de hospitalización; Pronóstico; Desnutrición.

\section{Introdução}

A análise dos aspectos nutricionais dos pacientes hospitalizados revela que estes apresentam necessidades alimentares especiais, determinadas, principalmente, pelos desequilíbrios metabólicos encerrados pela doença. Vários estudos, no Brasil e no mundo, já demonstraram que a desnutrição possui elevada prevalência entre os pacientes hospitalizados (Leite et al., 2005; Aquino \& Philippi, 2012).

A desnutrição em pacientes internados correlaciona-se com o aumento da frequência de intercorrências clínicas e mortalidade, além de também ser associada a um maior período de internamento hospitalar, maior risco de infecções e impacto negativo nos custos para o serviço de saúde. Sabe-se que a desnutrição em indivíduos hospitalizados decorre de uma gama de condições, prévias e posteriores à hospitalização, podendo ser atribuída à doença e/ou à terapia instituída (Aquino \& Philippi, 2011; Campelo et al., 2007).

A etiologia da desnutrição hospitalar é complexa. A doença subjacente pela qual o paciente é hospitalizado é um fator importante na apresentação de desnutrição, mas é errado considerar que a desnutrição é inerente à doença e, como tal, não tratável. Isto tem sido demonstrado em estudos de intervenção nutricional em diferentes patologias realizados por Elia et al. (2005) nos quais a desnutrição foi parcialmente reversível.

Apesar de não haver consenso acerca dos critérios diagnósticos de desnutrição, a avaliação do estado nutricional, bem como a identificação dos fatores de risco para desnutrição e a determinação de instrumentos de triagem nutricional são fundamentais para a adequada condução da terapêutica aplicada ao paciente (Aquino \& Philippi, 2011; Aquino \& Philippi, 2012). Em 1994, a ADA (American Dietetic Association) definiu como risco nutricional a "presença de fatores que podem acarretar e/ou agravar a desnutrição em pacientes".

O risco nutricional relaciona-se a vieses imbricados no estado geral do paciente, assim como na história da doença atual, agregando aspectos psicossociais e físicos à análise. Dessa maneira, no intuito de propiciar a terapia nutricional adequada para cada paciente, a identificação dos fatores de risco, a partir de critérios padronizados, é essencial ao tratamento global do paciente internado, elevando a eficácia da conduta instituída, considerando precocidade e coerência, que se reflete ao bem-estar ao doente (Maicá \& Schweigert, 2008; Aquino \& Philippi, 2011). Este componente está fortemente ligado à indicação inadequada de terapias nutricionais, avaliação inconsistente do estado nutricional e acompanhamento deficitário da resposta do paciente ao tratamento implementado (Leite et al., 2005). 
Dada a complicada rede de fatores relevantes na identificação e monitoração do estado nutricional do paciente hospitalizado, reconhece-se, atualmente, o impacto da formação e atuação de Equipe Multidisciplinar de Terapia Nutricional, formada por médicos, enfermeiros, nutricionistas e farmacêuticos, de modo a assegurar atenção adequada ao paciente em tratamento. Além dos agravos à saúde, tais como infecções, traumatismos, cirurgias e restrição hídrica e alimentar, a negligência da equipe assistente ao cuidado nutricional figura entre os elementos que mais comumente contribuem para o estabelecimento de quadros de desnutrição (Leite et al., 2005). Considerando o exposto, o objetivo desta pesquisa foi elaborar revisão sistemática sobre o efeito da avaliação nutricional sobre o tempo de internação hospitalar, caracterizando a prevalência de desnutrição e risco nutricional nos pacientes hospitalizados.

\section{Metodologia}

Realizou-se uma revisão sistemática sobre estudos originais, com análise secundária de dados. O levantamento bibliográfico de artigos sobre avaliação nutricional e seu efeito no tempo de internação hospitalar foi feito através dos bancos de dados eletrônicos LILACS, MEDLINE, SCIELO, PUBMED e BIBLIOTECA COCHRANE, no período de 2004 a 2014.

Foram utilizados os seguintes critérios de inclusão: a. Estudos publicados no período de Jan/2004 a Jan/2014; b. Publicações em idioma Inglês, Português e Espanhol; c. Estudos com objetivo principal ou secundário de analisar o efeito da avaliação nutricional em pacientes hospitalizados; e d. Estudos com população alvo com idade a partir de 18 anos. Os critérios de exclusão utilizados foram: a. Publicações na forma de Relatos e Séries de Casos, Teses, Dissertações, Anais de Congresso e Abstracts, Respostas ou Comentários de Artigos originais e Artigos de Revisão; b. Estudos que utilizaram pacientes cirúrgicos e/ou oncológicos ou gestantes; e c. Estudos envolvendo modelos animais.

A estruturação do banco de dados com os elementos de sistematização para a análise secundária de dados foi baseada no modelo preconizado por Figueiredo \& Tavares-Neto (2001); e busca nas bases de dados eletrônicas foi realizada por meio dos descritores relacionados ao objetivo principal: "Nutrition Assessment", "Length of Stay", "Prognosis".

\subsection{Etapas da seleção dos artigos}

a) Os artigos selecionados pela leitura do título foram avaliados separadamente, pelo pesquisador, segundo os critérios de inclusão, exclusão e duplicidade. Os resumos (Abstracts) foram avaliados por três revisores, independentes, segundo os critérios de inclusão e exclusão. As discordâncias foram submetidas à avaliação para inclusão ou exclusão, em consenso pelos revisores.

b) Resgate do artigo completo no Serviço Cooperativo de Acesso a Documentos - SCAD (BVS/BIREME http://scad.bvs.br) e em revistas disponíveis no Portal da CAPES (Coordenação de Aperfeiçoamento de Pessoal de Nível Superior - www.periódicos.capes.gov.br).

c) Leitura do artigo completo pelos revisores para seleção ou exclusão do artigo; As discordâncias foram submetidas à avaliação para inclusão ou exclusão, em consenso.

\subsection{Estratégia para síntese das informações}

Após a seleção final dos artigos, todos os trabalhos que satisfizeram os critérios de seleção foram lidos na íntegra em conjunto pelos autores. Foram indexados ao banco de dados as principais informações caracterizadoras de cada trabalho incluído, além de outros dados de relevância para a revisão. 


\subsection{Estratégia para elaboração descritiva das informações}

As informações sintetizadas e sistematizadas no banco de dados foram confrontadas, sendo criado um corpo de texto.

\subsection{Qualidade dos estudos}

Os artigos foram classificados utilizando uma adaptação do modelo idealizado por Figueiredo \& Tavares-Neto (2001). A partir da quantidade de variáveis presentes, utilizando a Ficha de Sistematização de Dados, os artigos foram classificados em:

- Classe A (excelente): $100 \%$ das variáveis;

- Classe B (ótima ou boa): de 76 a 99\% das variáveis;

- Classe C (regular): de 51 a 75\% das variáveis;

- Classe D (ruim): $50 \%$ ou menos das variáveis pesquisadas.

\subsection{Planejamento estatístico}

Após a coleta e seleção qualitativa dos dados dos artigos, os mesmos foram implantados em uma planilha e foi realizada análise descritiva.

\subsection{A busca dos artigos}

A busca dos artigos teve como ponto de partida dois braços de associação dos descritores. Um deles associou "Nutrition assessment" e "Length of stay" e o outro buscou artigos que associaram "Nutrition assessment" e "Prognosis". Realizou-se a busca nas bases de dados da Biblioteca Regional de Medicina (BIREME) / Biblioteca Virtual em Saúde (BVS), publicados no período de janeiro de 2004 a janeiro 2014.

Filtrou-se os estudos publicados nos idiomas espanhol, inglês e português, que avaliaram pacientes maiores de 18 anos, de ambos os gêneros. Foram recuperados 621 artigos identificados através do primeiro braço da pesquisa e 640 publicações por meio do segundo.

Aplicando-se como filtros de seleção o período e o idioma de publicação, além de estudos que envolviam apenas seres humanos, foram identificados 341 artigos no primeiro braço de busca e 332 estudos no segundo.

Dos 62 artigos estudados (textos completos), 10 estudos foram selecionados como adequados aos critérios de inclusão desta revisão (Figura 1). 
Figura 1. Fluxograma de seleção dos artigos.

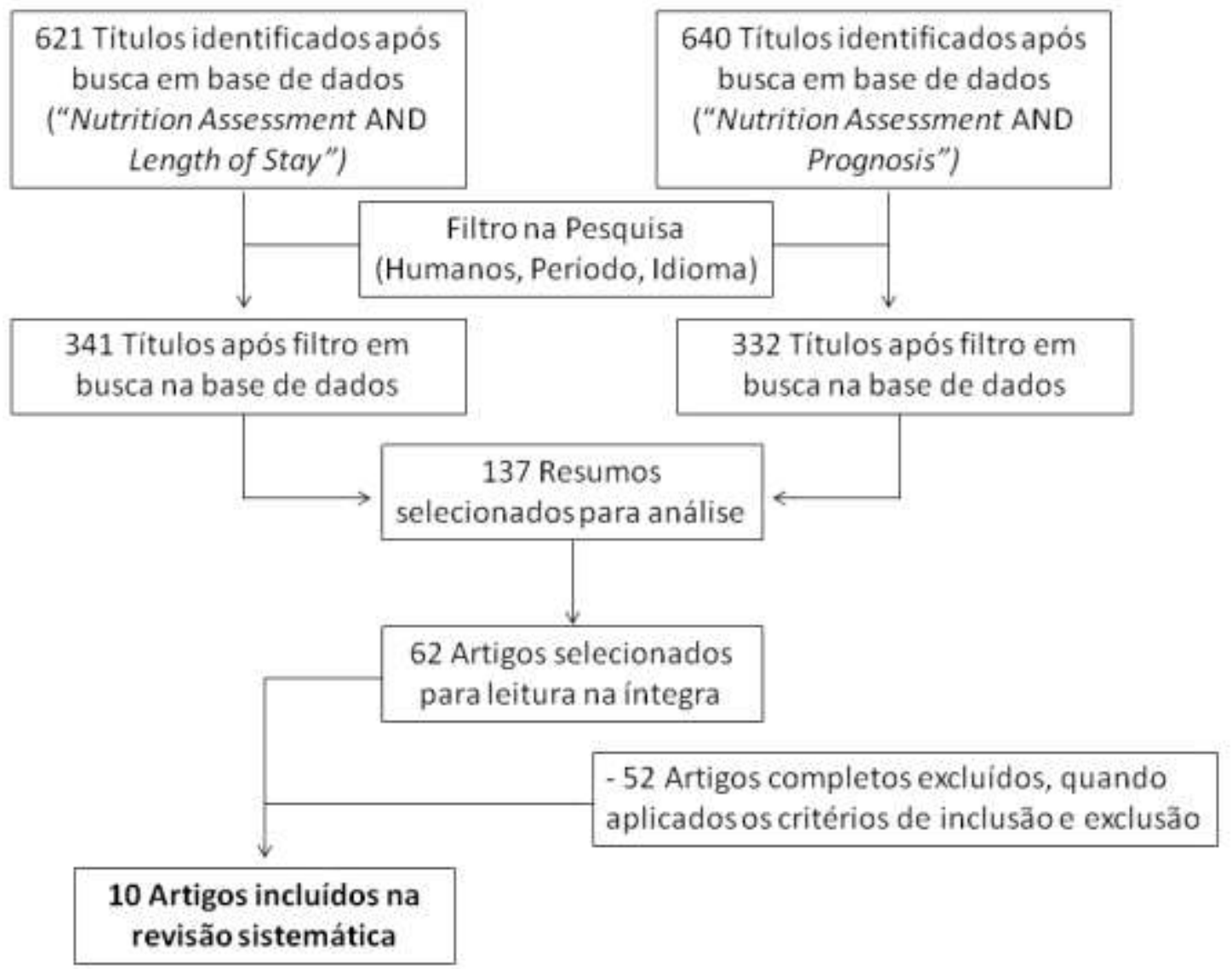

Fonte: Autores (2021).

Os artigos selecionados como relevantes para este estudo foram:

Artigo 1 - BAUER, J. M. et al. Comparison of the Mini Nutritional Assessment, Subjective Global Assessment, and Nutritional Risk Screening (NRS 2002) for nutritional screening and assessment in geriatric hospital patients. Zeitschrift für Gerontologie und Geriatrie, v. 38, n. 5, p. 322-327, 2005. ISSN 0948-6704.

Artigo 2 - FELDBLUM, I. et al. Nutritional risk and health care use before and after an acute hospitalization among the elderly. Nutrition, v. 25, n. 4, p. 415-420, 2009. ISSN 0899-9007.

Artigo 3 - GARIBALLA, S.; FORSTER, S. Malnutrition is an independent predictor of 1-year mortality following acute illness. British Journal of Nutrition, v. 98, n. 02, p. 332-336, 2007. ISSN 1475-2662.

Artigo 4 - LÓPEZ-GÓMEZ, J. J. et al. Valoración del riesgo nutricional en pacientes ancianos hospitalizados mediante diferentes herramientas. Endocrinología y Nutrición, v. 58, n. 3, p. 104-111, 2011. ISSN 1575-0922.

Artigo 5 - MARTINEAU, J. et al. Malnutrition determined by the patient-generated subjective global assessment is associated with poor outcomes in acute stroke patients. Clinical Nutrition, v. 24, n. 6, p. 1073-1077, 2005. ISSN 0261-5614.

Artigo 6 - RASLAN, M. et al. Complementarity of Subjective Global Assessment (SGA) and Nutritional Risk Screening 2002 (NRS 2002) for predicting poor clinical outcomes in hospitalized patients. Clinical Nutrition, v. 30, n. 1, p. 4953, Feb 2011. ISSN 0261-5614. Disponível em: <<Go to ISI>://WOS:000287902200008 >. 
Artigo 7 - RIQUELME, R. et al. Neumonía adquirida en la comunidad en el anciano hospitalizado: Aspectos clínicos y nutricionales. Revista Médica de Chile, v. 136, n. 5, p. 587-593, 2008. ISSN 0034-9887.

Artigo 8 - SANCHEZ-MUNOZ, L. A. et al. Mini nutritional assessment (MNA) as nutrition screening tool in internal medicine. Advantages and disadvantages. Revista Clinica Espanola, v. 210, n. 9, p. 429-437, Oct 2010. ISSN 0014-2565. Disponível em: < <Go to ISI >:/WOS:000283268800001 >.

Artigo 9 - STRATTON, R. J. et al. 'Malnutrition Universal Screening Tool'predicts mortality and length of hospital stay in acutely ill elderly. British Journal of Nutrition, v. 95, n. 02, p. 325-330, 2006. ISSN 1475-2662.

Artigo 10 - THOMAS, J.; ISENRING, E.; KELLETT, E. Nutritional status and length of stay in patients admitted to an Acute Assessment Unit. Journal of Human Nutrition and Dietetics, v. 20, n. 4, p. 320-328, 2007. ISSN 1365-277X.

\section{Resultados}

Dos artigos identificados e após avaliação dos revisores, excluindo-se os trabalhos repetidos entre os mesmos e as discordâncias consensuais, a busca dos artigos resultou no número de 137, os quais tiveram os 'Resumos' ou 'Abstracts' avaliados pelos investigadores. Desses 137 artigos, 62 trabalhos foram selecionados para terem seus textos completos estudados.

\subsection{A qualidade dos estudos}

Dos artigos selecionados foram analisadas as variáveis sistematizadas, conforme os critérios estabelecidos por Figueiredo \& Tavares-Neto (2001). A consolidação dessa análise está representada na Tabela 1.

Tabela 1. Classificação dos artigos conforme a qualidade do estudo.

\begin{tabular}{lcc}
\hline QUALIDADE DA DESCRIÇão & $\mathbf{n}$ & \% \\
\hline Classe A (Excelente) & 00 & 00 \\
Classe B (Ótima ou Boa) & 09 & 90 \\
Classe C (Regular) & 01 & 10 \\
Classe D (Ruim) & 00 & 00 \\
TOTAL & $\mathbf{1 0}$ & $\mathbf{1 0 0}$ \\
\hline
\end{tabular}

Legenda: $\mathrm{n}$ - número de artigos.

Fonte: Autores (2021).

\subsection{A origem dos estudos}

A origem dos estudos selecionados mostrou-se bastante variada, rendendo artigos nacionais e internacionais. A Tabela 2 sintetiza essa diversidade.

Tabela 2. Frequência dos artigos segundo o local de origem do estudo.

\begin{tabular}{lcc}
\hline LOCAL DO ESTUDO & n & $\%$ \\
\hline Alemanha & 1 & 10 \\
Austrália & 2 & 20 \\
Brasil & 1 & 10 \\
Chile & 1 & 10 \\
Israel & 1 & 10 \\
México & 2 & 20 \\
Reino Unido & 2 & 20 \\
TOTAL & $\mathbf{1 0}$ & $\mathbf{1 0 0}$ \\
\hline
\end{tabular}




\subsection{O tipo de estudo}

Sob o aspecto metodológico do tipo de estudo, a maioria dos artigos selecionados aborda o assunto de maneira prospectiva. Comparativamente, a Tabela 3 apresenta os estudos analisados.

Tabela 3. Classificação dos artigos conforme o tipo de estudo.

\begin{tabular}{lc}
\hline ESTUDO & TIPO DE ESTUDO \\
\hline Feldblum et al., 2009 & Prospectivo \\
Riquelme et al., 2008 & Prospectivo \\
Martineau et al., 2005 & Retrospectivo \\
Raslan et al., 2011 & Prospectivo \\
López-Gómez et al., 2011 & Retrospectivo \\
Sanchez-Munoz et al., 2010 & Prospectivo \\
Thomas et al., 2007 & Prospectivo \\
Stratton et al., 2006 & Prospectivo \\
Bauer et al., 2005 & Prospectivo \\
Gariballa \& Forster, 2007 & Prospectivo \\
\hline
\end{tabular}

Fonte: Autores (2021).

\subsection{O periódico de publicação}

Houve grande diversidade no resgate dos artigos em função do periódico no qual foi publicado. A Tabela 4 apresenta bem essa variedade.

Tabela 4. Frequência dos artigos de acordo com o periódico de publicação.

\begin{tabular}{lc}
\hline PERIÓDICO & n \\
\hline British Journal of Nutrition & 2 \\
Clinical Nutrition & 2 \\
Zeitschrift Fur Gerontologie Geriatrie & 1 \\
Revista médica de Chile & 1 \\
Nutrition & 1 \\
Endocrinología y Nutrición & 1 \\
Journal of Human Nutrition and Dietetics & 1 \\
Revista Clínica Española & 1 \\
TOTAL & $\mathbf{1 0}$ \\
\hline
\end{tabular}

Legenda: $\mathrm{n}$ - número de artigos.

Fonte: Autores (2021).

\subsection{Quantidade de pacientes envolvidos nos estudos}

Tomando-se o total de pacientes envolvidos nos estudos selecionados, a população acompanhada somou 2.181 pacientes, todos com idade superior a 18 anos. 


\subsection{A idade e o gênero dos pacientes nos estudos analisados}

Considerando a faixa etária dos pacientes estudados, alguns relatos não especificaram intervalos de idade da população acompanhada. Isto é, registrou-se apenas que o grupo estudado apresentava mais de 18 anos. Outros, entretanto, especificaram grupos etários, cuja consolidação da análise de tais estudos permitiu o enquadramento nos seguimentos apresentados abaixo (Tabela 5). A média de idade dos pacientes estudados está representada na Tabela 6. O Artigo 6, selecionado para esta revisão, não mencionou dados sobre a idade dos pacientes estudados.

A proporção de pacientes em função do gênero, assim como a idade, não foi especificada em alguns estudos, tendo sido diferenciada em outros (Tabela 7).

Tabela 5. Frequência dos pacientes conforme a faixa etária.

\begin{tabular}{ccc}
\hline IDADE & $\mathbf{N}$ & $\mathbf{\%}$ \\
\hline$\geq 18$ anos. Sem intervalo especificado. & 842 & 38,6 \\
$18-59$ anos & 91 & 4,2 \\
$\geq 60$ anos & 1.248 & 57,2 \\
TOTAL & $\mathbf{2 . 1 8 1}$ & $\mathbf{1 0 0}$ \\
\hline
\end{tabular}

Legenda: $\mathrm{N}$ - número de pacientes.

Fonte: Autores (2021).

Tabela 6. Média de idade dos pacientes.

\begin{tabular}{ccc}
\hline ARTIGO & MÉDIA DE IDADE (anos) & DP \\
\hline Artigo 1 & 80,20 & $\pm 7,7$ \\
Artigo 2 & 73,80 & $\pm 5,6$ \\
Artigo 3 & 76,00 & $\pm 6,0$ \\
Artigo 4 & 80,86 & $\pm 4,2$ \\
Artigo 5 & 72,00 & $\pm 12,9$ \\
Artigo 6 & $*$ & \\
Artigo 7 & 63,00 & $\pm 19,0$ \\
Artigo 8 & 81,00 & $\pm 7,0$ \\
Artigo 9 & 85,00 & $\pm 5,5$ \\
Artigo 10 & 79,90 & $\pm 11,0$ \\
\hline
\end{tabular}

Legenda: DP - Desvio padrão.

Fonte: Autores (2021).

Tabela 7. Frequência dos pacientes em função do gênero.

\begin{tabular}{lcc}
\hline \multicolumn{1}{c}{ GẾNERO } & $\mathbf{N}$ & \% \\
\hline Sem especificação & 1.188 & 54,5 \\
Masculino & 511 & 23,4 \\
Feminino & 482 & 22,1 \\
TOTAL & $\mathbf{2 . 1 8 1}$ & $\mathbf{1 0 0}$ \\
\hline
\end{tabular}

Legenda: $\mathrm{N}$ - número de pacientes.

Fonte: Autores (2021). 


\subsection{Avaliação nutricional dos pacientes estudados}

Em função da prevalência de Desnutrição e/ou do Risco Nutricional dos pacientes acompanhados, distinção feita conforme o instrumento de avaliação nutricional aplicado em cada estudo, a Tabela 8 evidencia o resultado da avaliação nutricional realizada em cada trabalho.

Tabela 8. Prevalência de desnutrição e/ou risco nutricional dos pacientes estudados, de acordo com o instrumento de avaliação nutricional aplicado.

\begin{tabular}{|c|c|c|}
\hline ESTUDO & EM RISCO NUTRICIONAL & DESNUTRIDOS \\
\hline Feldblum et al., 2009 & $37,72 \%$ & $0,98 \%$ \\
\hline Riquelme et al., 2008 & - & $69,9 \%$ \\
\hline Martineau et al., 2005 & - & $19,2 \%$ \\
\hline Raslan et al., 2011 & $27,9 \%$ & $38,9 \%$ \\
\hline \multirow{2}{*}{ López-Gómez et al., 2011} & NRI $96,3 \%$ & - \\
\hline & GNRI $81,3 \%$ & \\
\hline Sanchez-Munoz et al., 2010 & $36,8 \%$ & $4,7 \%$ \\
\hline Thomas et al., 2007 & - & $53 \%$ \\
\hline Stratton et al., 2006 & $58 \%$ & - \\
\hline \multirow{2}{*}{ Bauer et al., 2005} & MNA 37,5\%; & MNA $32,5 \%$ \\
\hline & NRS2002 63,8\% & SGA $45 \%$ \\
\hline Gariballa \& Forster, 2007 & - & - \\
\hline
\end{tabular}

Legenda: GNRI - Geriatric Nutritional Risk Index; NRI - Nutritional Risk Index; NRS 2002 - Nutritional Risk Screening 2002; MNA - Mini Nutritional Assessment; SGA - Subjective Global Assessment.

Fonte: Autores (2021).

\subsection{Instrumentos de avaliação nutricional}

Entre os estudos selecionados, houve importante diversidade entre os instrumentos de avaliação nutricional aplicados.

A Tabela 9 evidencia essa distinção especificando a ferramenta utilizada em cada um dos estudos analisados.

Tabela 9. Instrumentos de avaliação nutricional utilizados nos estudos selecionados.

\begin{tabular}{|c|c|c|c|c|c|c|c|c|c|}
\hline \multirow{2}{*}{ AUTOR } & \multicolumn{9}{|c|}{ INSTRUMENTOS DE AVALIAÇÃO NUTRICIONAL } \\
\hline & MUST & MNA & SGA & PG-SGA & NRS 2002 & NRI & GNRI & Bioquímicas & Antropometria \\
\hline Martineau et al., 2005 & & & & $\mathrm{X}$ & & & & $\mathrm{X}$ & \\
\hline Bauer et al., 2005 & & $\mathrm{X}$ & $\mathrm{X}$ & & $\mathrm{X}$ & & & $\mathrm{X}$ & \\
\hline Stratton et al., 2006 & $\mathrm{X}$ & & & & & & & & \\
\hline Gariballa \& Forster, 2007 & & & & & & & & $\mathrm{X}$ & $\mathrm{X}$ \\
\hline Thomas et al., 2007 & & & $\mathrm{X}$ & & & & & $\mathrm{X}$ & \\
\hline Riquelme et al., 2008 & & & & & & & & $\mathrm{X}$ & $\mathrm{X}$ \\
\hline Feldblum et al., 2009 & & $\mathrm{X}$ & & & & $\mathrm{X}$ & & $\mathrm{X}$ & \\
\hline Sanchez-Munoz et al., 2010 & & $\mathrm{X}$ & & & & & & $\mathrm{X}$ & \\
\hline Raslan et al., 2011 & & & $\mathrm{X}$ & & $\mathrm{X}$ & & & & \\
\hline López-Gómez et al., 2011 & & & & & & $\mathrm{X}$ & $\mathrm{X}$ & $\mathrm{X}$ & \\
\hline
\end{tabular}

Legenda: GNRI - Geriatric Nutritional Risk Index; NRI - Nutritional Risk Index; NRS 2002 - Nutritional Risk Screening 2002; MNA - Mini Nutritional Assessment; MUST - Malnutrition Universal Screening Tool; PG-SGA - Patient-Generated Subjective Global Assessment; SGA - Subjective Global Assessment; *Albumina, Pré-albumina, Ferritina e Contagem leucocitária.

Fonte: Autores (2021). 


\subsection{Associação desnutrição e tempo de internação hospitalar}

A associação entre o estado ou o risco nutricional e tempo de internação hospitalar foi estudada comparando pacientes bem nutridos com os grupos em risco de desnutrição ou desnutridos. Tais dados, conforme os estudos selecionados são evidenciados na Tabela 10 .

Tabela 10. Comparação do tempo de internação hospitalar entre os grupos de pacientes considerados bem nutridos e os que se apresentavam desnutridos ou em risco de desnutrição.

\begin{tabular}{|c|c|c|c|c|}
\hline ESTUDO & $\begin{array}{c}\text { BEM } \\
\text { NUTRIDOS }\end{array}$ & MAL NUTRIDOS & RISCO NUTRICIONAL & $\mathbf{P}$ \\
\hline Feldblum et al., 2009 & 5 dias $( \pm 0,4)$ & ------- & 7,14 dias $( \pm 0,8)$ & 0,01 \\
\hline Riquelme et al., 2008) & $11 \operatorname{dias}( \pm 6)$ & 13,7 dias $( \pm 10,5)$ & -------- & 0,024 \\
\hline Martineau et al., 2005 & 8 dias (2-33) & 13 dias (4-55) & -------- & $<0,001$ \\
\hline Raslan et al., 2011 & \multicolumn{4}{|c|}{ 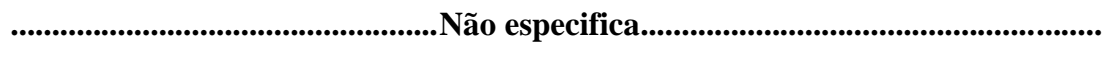 } \\
\hline López-Gómez et al., 2011 & \multicolumn{4}{|c|}{ Não especifica } \\
\hline Sanchez-Munoz et al., 2010 & \multicolumn{3}{|c|}{11,08 dias $( \pm 9,79)^{1}$} & ----- \\
\hline Thomas et al., 2007 & 4 dias $(2-19)$ & 5 dias $(1-24)$ & ------ & 0,323 \\
\hline Stratton et al., 2006 & \multicolumn{4}{|c|}{$\begin{array}{l}\text { Médio: } 24 \text { dias (16-32) } \\
\text { Alto: } 28 \text { dias (21-35) }\end{array}$} \\
\hline Bauer et al., 2005 & $3,9 \operatorname{dias}( \pm 7,8)$ & ------- & $\begin{array}{r}\text { Moderado 5,4 dias }( \pm 8,2) \\
\text { Severo } 10,8 \text { dias }( \pm 26,3)\end{array}$ & $<0,001$ \\
\hline Gariballa \& Forster, 2007 & \multicolumn{4}{|c|}{ 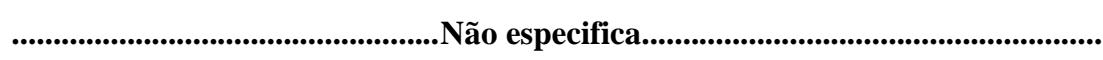 } \\
\hline
\end{tabular}

Legenda: $\mathrm{p}$ - Probabilidade de significância; ${ }^{1}$ - Média de tempo de internação de todos os pacientes do referido estudo, sem distinguir grupo em relação ao estado ou risco nutricional.

Fonte: Autores (2021).

\section{Discussão}

O diagnóstico nutricional, mediante critérios fidedignos e de relevância reconhecida pela ciência, representa um passo importante para a terapêutica intra-hospitalar, bem como se mostra eficiente para abreviar o período de internação, resultando em melhora clínica significativa dos pacientes assistidos. Dessa forma, estabelecer esse tipo de propedêutica e abordagem, de modo sistemático e uniforme num dado serviço de referência em assistência à saúde, definindo o perfil nutricional do indivíduo que se hospitaliza, pode contribuir grandemente para a efetividade do atendimento proposto, bem como se refletir em bem-estar do paciente.

Em estudo prospectivo de coorte, que incluiu 818 pacientes triados através da SGA, evidenciou que cerca de $29 \%$ dos pacientes admitidos no serviço estavam desnutridos, apresentaram maior tempo de internação hospitalar e eram mais propensos a serem readmitidos após 15 dias da alta. Além disso, o mesmo estudo mostrou ainda que a desnutrição foi um preditor significativo de mortalidade e a assistência prestada a tais pacientes requerido maior demanda de recursos financeiros da instituição de saúde (Lim et al., 2012).

Assim, a desnutrição propriamente dita pode, em suma, ser predisposta por múltiplos fatores, incluindo ingesta inadequada de alimentos previamente à internação hospitalar, comorbidades crônicas, idade, uso de medicamentos, depressão e distúrbios da função cognitiva. Tendo em vista esses elementos, a identificação precoce do estado e de fatores de risco 
nutricionais do paciente admitido no serviço de saúde, pode permitir melhores resultados clínicos, aumentando a eficácia de ações terapêuticas e profiláticas em relação a condição nutricional e geral do paciente (Feldblum et al., 2009).

Um outro estudo, este observacional, transversal e multicêntrico, mostrou uma prevalência de desnutridos nos seus pacientes assistidos em torno de $23 \%$, tendo a desnutrição sido associada ao aumento no tempo de internação hospitalar e a maiores custos na assistência prestada (Álvarez-Hernández et al., 2012).

De acordo com dados do Inquérito Brasileiro de Avaliação Nutricional Hospitalar (IBRANUTRI), realizado com 4000 pacientes adultos hospitalizados, há uma elevada prevalência de desnutrição nos hospitais brasileiros $(48,1 \%$ de desnutrição na população estudada) [Raslan et al., 2011]. O Estudo Latino Americano de Nutrição (ELAN) aplicado em hospitais gerais de treze países da América Latina, com uma amostra de 9348 pacientes adultos, corroborou com tais evidências, identificando 50,2\% de desnutrição (Crestani et al., 2011). Já no estudo epidemiológico, transversal, de Rezende et al. (2004), que avaliou o estado nutricional de 244 doentes internados em uma instituição filantrópica em Salvador (Bahia), revelou-se que mais da metade $(63,11 \%)$ dos pacientes hospitalizados encontram-se desnutridos.

Esta revisão identificou nos estudos avaliados uma prevalência de desnutrição à admissão hospitalar que variou entre 0,98\% e 69,9\% (Tabela 8) [Riquelme et al. 2008; Feldblum et al., 2009]. No entanto, apesar do extenso intervalo de variação mencionado, observa-se que este índice permanece de acordo com o que foi evidenciado em estudos anteriores. Essa ampla variação pode ser explicada pela diversidade de ferramentas para avaliação nutricional, como também em virtude do perfil socioeconômico do paciente estudado (Kyle et al., 2005).

Ainda considerando a elevada prevalência da desnutrição à admissão hospitalar, esta revisão comprovou o interesse pelo estudo do tema ao redor do mundo, uma vez que os trabalhos publicados foram realizados em diversos países. Os dez artigos selecionados apresentam sete nacionalidades diferentes, tendo a Austrália, o México e o Reino Unido figurado com dois artigos cada um (Tabela 2).

Com relação a idade dos pacientes, esta revisão incluiu estudos que envolveram indivíduos com mais de 18 anos. Observou-se que a maioria deles $(57,2 \%)$ possuía idade igual ou superior a 60 anos e 38,6\% da população estudada não teve enquadramento em intervalo etário (Tabela 5). No entanto, a análise descritiva dos estudos selecionados nesta revisão mostra que média de idade variou de 63 a 85 anos (Tabela 6). Ou seja, dentre as faixas etárias categorizada, a população idosa (idade $\geq$ 60 anos) merece cuidado em relação ao estado nutricional, pois além da significância quantitativa de pacientes em estado de desnutrição ou de risco nutricional, este grupo apresenta maiores flutuações metabólicas e de composição corporal, estando, portanto, mais sensíveis à desnutrição, aumentando o risco de desfechos clínicos desfavoráveis, o tempo de internação hospitalar e a mortalidade geral (López-Gómez et al., 2011).

Sob o aspecto de gênero, o Artigo 2 evidenciou um número maior de homens no grupo de pacientes bem nutridos, com significância estatística ( $\mathrm{p}=0,02)$ (Feldblum et al., 2009). Em contrapartida, o Artigo 9 apresentou dados mostrando que não houve diferença significativa entre os gêneros em relação ao risco de desnutrição (Stratton et al., 2006).

Os estudos desta revisão utilizaram diversos instrumentos para a avaliação nutricional. No total foram sete modelos diferentes (MUST, MNA, SGA, PG-SGA, NRS 2002, NRI e GNRI), além de variáveis antropométricas e bioquímicas (Tabela 9). Apenas dois dos dez estudos não utilizaram dados bioquímicos na avaliação nutricional. Os modelos mais utilizados foram o MNA e o SGA.

A variedade de ferramentas que podem ser usadas para determinar o estado nutricional do paciente aumenta a possibilidade de discrepâncias entre resultados das avaliações, já que os mais variados critérios ou variáveis podem ser utilizados (antropometria, bioquímica, exame físico, avaliação subjetiva) (Kyle et al., 2005). A comparação entre tais variáveis conforme o instrumento de avaliação nutricional utilizado em cada estudo selecionado nesta revisão encontra-se evidenciado na Tabela 11. 
Os métodos podem avaliar o grau de desnutrição ou o risco de desnutrição no ambiente hospitalar. Algumas ferramentas não dão alternativas quando certas variáveis não podem ser avaliadas, como peso e altura em idosos e/ou acamados. O MUST, por exemplo, contorna essa dificuldade (Stratton et al., 2006).

Além do MNA e do GNRI, que proporciona uma avaliação direcionada à população idosa, o MUST também apresenta vantagens para esse grupo, pois oferece alternativas em sua avaliação no que diz respeito a medidas antropométricas (peso, altura e IMC). O MUST foi desenvolvido para avaliar todos os adultos e pode ser usado mesmo quando não é possível a medida da altura e do peso, o que não ocorre nos outros instrumentos de avaliação nutricional (Stratton et al., 2006), como NRS-2002, MNA e SGA.

Tabela 11. Comparação entre as variáveis utilizadas pelos instrumentos de avaliação nutricional.

\begin{tabular}{lccccccc}
\hline \multicolumn{1}{c}{ VARIÁVEL } & MUST & NRS-2002 & MNA & SGA & PG-SGA & NRI & GNRI \\
\hline IMC & $\mathrm{X}$ & $\mathrm{X}$ & $\mathrm{X}$ & $\mathrm{X}$ & & & \\
Perda de peso & $\mathrm{X}$ & $\mathrm{X}$ & $\mathrm{X}$ & & $\mathrm{X}$ & $\mathrm{X}$ & $\mathrm{X}$ \\
$\begin{array}{l}\text { Efeito de } \\
\text { doença aguda }\end{array}$ & $\mathrm{X}$ & $\mathrm{X}$ & $\mathrm{X}$ & $\mathrm{X}$ & $\mathrm{X}$ & & \\
$\begin{array}{l}\text { Diminuição da } \\
\text { ingesta alimentar }\end{array}$ & & $\mathrm{X}$ & $\mathrm{X}$ & $\mathrm{X}$ & $\mathrm{X}$ & & \\
$\begin{array}{l}\text { Sintomas gastrointestinais } \\
\text { Capacidade funcional }\end{array}$ & & & & $\mathrm{X}$ & $\mathrm{X}$ & & \\
$\begin{array}{l}\text { Idade > 70 anos } \\
\text { Albumina Sérica }\end{array}$ & & $\mathrm{X}$ & & $\mathrm{X}$ & $\mathrm{X}$ & & \\
Mobilidade & & & $\mathrm{X}$ & & & & $\mathrm{X}$ \\
Problemas neurofisiológicos & & & $\mathrm{X}$ & & & & \\
Exame físico & & & & $\mathrm{X}$ & $\mathrm{X}$ & & \\
\hline
\end{tabular}

Legenda: GNRI - Geriatric Nutritional Risk Index; NRI - Nutritional Risk Index; NRS 2002 - Nutritional Risk Screening 2002; MNA - Mini Nutritional Assessment; MUST - Malnutrition Universal Screening Tool; PG-SGA - Patient-Generated Subjective Global Assessment; SGA - Subjective Global Assessment.

Fonte: Araújo et al. (2021).

Dentre os parâmetros bioquímicos, a quantificação da Albumina sérica pode ser útil quando analisada cuidadosamente. A hipoalbuminemia pode estar relacionada a estados de desnutrição, principalmente crônicos, devido sua meia-vida longa (20 dias). Relatos indicam que o nível de albumina sérica é um bom preditor de tempo de internação hospitalar; a redução do seu nível à admissão está associada a um aumento no tempo de hospitalização, sendo também um preditor de morbi-mortalidade (Dias et al., 2011).

Entretanto, o Artigo 1 selecionado nesta revisão, foi o único a demonstrar correlação estatisticamente significativa entre o nível de albumina sérica e o tempo de internação da população estudada, tendo como método de avaliação nutricional o MNA (Bauer et al., 2005). Os Artigos 5 e 10 desta seleção, em contrapartida, não evidenciaram correlação significativa entre níveis reduzidos de albumina no soro e o aumento do tempo de hospitalização (Martineau et al., 2005; Thomas et al., 2007).

O Artigo 2 segue nessa mesma linha evidenciando que não houve diferença nos níveis séricos de albumina entre os grupos; no entanto, os valores de colesterol sérico e hemoglobina foram significativamente menores no grupo de risco nutricional (Feldblum et al., 2009). Um dado deveras relevante acerca da eficiência da albumina sérica em predizer quaisquer desfechos clínicos é que ela sofre influência de fatores não nutricionais como sepse, trauma, inflamação ou doença hepática, constituindose como um marcador impreciso quando tomado isoladamente (Martineau et al., 2005). 
Assim sendo, em circunstâncias de descompensação aguda da condição de base, as respostas neuroendócrinas e metabólicas induzem a reorientação da síntese proteica priorizando as proteínas de fase aguda em detrimento da síntese de albumina, esclarecendo parcialmente a imprecisão da dosagem sérica dessa substância como marcador de avaliação nutricional (Gariballa \& Forster, 2007).

Além da albumina sérica, outro marcador laboratorial que pode ser utilizado para avaliação nutricional é a contagem total de linfócitos, cujo valor pode ser útil na mensuração do risco de complicações infecciosas em idosos, mas não é considerado bom método de avaliação nutricional isolado (Dias et al., 2011).

Analisando a associação entre desnutrição e tempo de internação hospitalar, a primeira progride à medida que aumenta o período de estadia na unidade de saúde: $46 \%$ dos pacientes já estão desnutridos nos primeiros cinco dias. Esse número cresce para $68 \%$ entre cinco e quinze dias e aumenta para $83 \%$ nos doentes internados há mais de quinze dias (Rezende et al., 2004).

Dos dez estudos selecionados nesta revisão, seis avaliaram a variação no tempo de internação e sua relação com o grau de desnutrição do paciente assistido. Observou-se que houve um aumento no tempo de permanência hospitalar dos que foram classificados como desnutridos ou com risco aumentado de desnutrição. A estadia hospitalar aumentou proporcionalmente ao grau de desnutrição dos pacientes internados. No entanto, conforme o Artigo 10, os pesquisadores não encontraram associação estatisticamente significativa entre a desnutrição e o aumento do tempo de internação ( $\mathrm{p}=0,323)$, como salientado na Tabela 10.

Um estudo prospectivo britânico, que envolveu 150 pacientes, evidenciou que o tempo de internação hospitalar foi progressivamente maior conforme o risco nutricional do paciente: os de baixo risco permaneceram cerca de 15 dias (IC=95\%), os de médio risco, 24 dias (IC=95\%) e os de alto risco contabilizaram 28 dias de hospitalização (IC=95\%) (Stratton et al., 2006). Bauer et al. (2005) verificaram que, dos 121 pacientes estudados, apenas o MNA mostrou associação significativa (Teste de Kruskal-Wallis, $\mathrm{p}=0$,044) entre o tempo de internação hospitalar e a avaliação nutricional realizada; constatando, assim, que associação semelhante não pode ser estabelecida ao se considerar o SGA (p=0,130) ou o NRS 2002 (p=0377).

Estudos acenam que a assistência ao paciente desnutrido ou em risco nutricional pode resultar em custos gerais elevados à unidade de saúde, apesar de nenhum dos artigos selecionados nesta revisão, referenciarem valores a esse respeito. Assim sendo, otimizar o cuidado frente a tais grupos populacionais, através de métodos sistemáticos e de simples aplicação, pode resultar em qualidade da assistência em saúde.

A avaliação nutricional é de fundamental importância não somente para uma adequada terapia nutricional, mas também serve como parâmetro para predizer desfechos clínicos.

A prevalência de desnutrição mostra-se elevada nos pacientes hospitalizados. Entretanto, de modo geral, existe um desconhecimento por parte da equipe de saúde com relação ao estado nutricional dos pacientes assistidos, influenciando de forma significativa e independente o tempo de permanência hospitalar, predispondo agravos e desfechos clínicos desfavoráveis.

Ferramentas de avaliação nutricional utilizam diversas variáveis clínicas e as evidências mostram que não existe um modelo ideal de avaliação nutricional no ambiente hospitalar. Logo, o instrumento a ser utilizado deverá ser baseado no perfil dos pacientes, na experiência dos profissionais e no tipo de instituição de saúde. No entanto, independente do modelo adotado, a avaliação nutricional deverá sempre ser realizada e, notadamente, o mais precocemente possível.

\section{Conclusão}

Conclui-se a partir desta revisão que pacientes com alto risco nutricional e/ou desnutrição no momento da hospitalização apresentam maior tempo de internação, evidenciando, portanto, a importância da avaliação nutricional à admissão hospitalar.

Novos estudos são necessários para avaliar e buscar um método prático e eficaz em determinar, de forma precisa, o estado nutricional de pacientes hospitalizados. Essa providência deverá contribuir para elucidar de modo mais claro a relação 
entre a desnutrição e/ou o risco nutricional e o tempo de internação hospitalar, melhorando o nível de assistência da equipe de saúde.

\section{Referências}

Álvarez-Hernández, J., Vila, M. P., León-Sanz, M., Lorenzo, G., Celaya-Pérez, S., García-Lorda, P., Araujo, K \& Guerri, B. S. (2012). Prevalence and costs of malnutrition in hospitalized patients; the PREDyCES Study. Nutrición Hospitalaria, 27(4), 1049-1059. http://www.nutricionhospitalaria.com/pdf/5986.pdf

Aquino, R. C. \& Philippi, S. T. (2011). Identificação de fatores de risco de desnutrição em pacientes internados. Revista da Associação Médica Brasileira, 57(6), 637-643. https://www.scielo.br/pdf/ramb/v57n6/v57n6a09.pdf

Aquino, R. C., Philippi, S. T. (2012). Desenvolvimento e avaliação de instrumentos de triagem nutricional. Revista Brasileira de Enfermagem, 65(4), 607-613. https://www.scielo.br/pdf/reben/v65n4/a09v65n4.pdf

Bauer, J. M., Vogl, T., Wicklein, S., Trögner, J., Mühlberg, W. \& Sieber, C. C. (2005). Comparison of the Mini Nutritional Assessment, Subjective Global Assessment, and Nutritional Risk Screening (NRS 2002) for nutritional screening and assessment in geriatric hospital patients. Zeitschrift für Gerontologie und Geriatrie, 38(5), 322-327.

Campelo, D. E., Vasconcelos, R. C. \& Cabral, M. A. (2007). Terapia Nutricional. Em: Condutas em Clínica Médica. Filgueira N. A. et al. $4^{\text {a }}$ ed. Rio de Janeiro: Guanabara Koogan, p. 759-771.

Crestani, N., Bieger, P., Kik, R. M. E., Dias, R. L., Alscher, S. \& Liener, R. S. C. (2011). Perfil nutricional de pacientes adultos e idosos admitidos em um hospital universitário. Ciência \& Saúde, 4(2), 45-49. https://doi.org/10.15448/1983-652X.2011.2.8520.

Dias, M. C. G., van Aanholt, D. P. J., Catalani, L. A., Rey, J. S. F., Gonzales, M.C., Coppini, L., Franco Filho, J. W., Paes-Barbosa, M. R., Horie, L., Abrahão, V. \& Martins, C. (2011). Triagem e Avaliação do Estado Nutricional. Associação Médica Brasileira e Conselho Federal de Medicina, 1-16. https://diretrizes.amb.org.br/_BibliotecaAntiga/triagem_e_avaliacao_do_estado_nutricional.pdf

Elia, M., Zellipour, L. \& Stratton, R. (2005). To screen or not to screen for adult malnutrition? Clinical Nutrition, 24(6), 867-884. DOI:10.1016/j.clnu.2005.03.004.

Feldblum, I., German, L., Bilenko, N. Shahar, A., Enten, R., Greenberg, D., Harman, I., Castel, H \& Shahar, D. R. (2009). Nutritional risk and health care use before and after an acute hospitalization among the elderly. Nutrition, 25(4), 415-420. 10.1016/j.nut.2008.10.016

Figueiredo, G. C. \& Tavares-Neto, J. (2001). Estruturação de um banco de dados para análise secundária de informações em relatos ou série de casos. Revista Brasileira de Ortopedia, 36(11), 407-411. https://www.scielo.br/pdf/ag/v52n3/0004-2803-ag-52-03-239.pdf

Gariballa, S. \& Forster, S. (2007). Malnutrition is an independent predictor of 1-year mortality following acute illness. British Journal of Nutrition, 98(2), 332336. 10.1017 / S0007114507701708

Kyle, U. G., Genton, L. \& Pichard, C. (2005). Hospital length of stay and nutritional status. Current Opinion in Clinical Nutrition \& Metabolic Care, 8(4), 397402. 10.1097/01.mco.0000172579.94513.db.

Leite, H. P., Carvalho, W. B., Santana e Menezes, J. F. (2005). Atuação da equipe multidisciplinar na terapia nutricional de pacientes sob cuidados intensivos. Revista de Nutrição, 18(6), 777-784. https://www.scielo.br/pdf/rn/v18n6/a08v18n6.pdf

Lim, S. L., Ong, K. C. B., Chan, Y. H., Loke, W. C., Ferguson, M. \& Daniels, L. (2012). Malnutrition and its impact on cost of hospitalization, length of stay, readmission and 3-year mortality. Clinical Nutrition, 31(3), 345-350. 10.1016/ j.clnu.2011.11.001

López-Gómez, J. J., Calleja-Fernández, A., Ballesteros-Pomar, M. D., Vidal-Casariego, A., Brea-Laranjo, C., Fariza-Vicente, E., Arias-García, R. M. \& CanoRodríguez, I. (2011). Valoración del riesgo nutricional en pacientes ancianos hospitalizados mediante diferentes herramientas. Endocrinología y Nutrición, 58(3), 104-111. 10.1016/j.endonu.2011.01.002

Maicá, A. O. \& Schweigert, I. D. (2008). Avaliação nutricional em pacientes graves. Revista Brasileira de Terapia Intensiva, 20(3), 286-295. https://www.scielo.br/pdf/rbti/v20n3/v20n3a12.pdf

Martineau, J., Bauer, J. D., Isenring, E. \& Cohen, S. (2005). Malnutrition determined by the patient-generated subjective global assessment is associated with poor outcomes in acute stroke patients. Clinical Nutrition, 24(6), 1073-1077. 10.1016 / j.clnu.2005.08.010

Raslan, M., Gonzalez, M. C., Torrinhas, R. S. M. M., Ravacci, G. R., Pereira, J. C. R. \& Waitzberg, D. L. (2011). Complementarity of subjective global assessment (SGA) and nutritional risk screening 2002 (NRS 2002) for predicting poor clinical outcomes in hospitalized patients. Clinical Nutrition, 30(1), 4953. $10.1016 /$ j.clnu.2010.07.002

Rezende, I. F. B., Oliveira, V. S., Kuwano, E. A., Leite, A. P. B., Rios, I., Dórea, Y. S. S. \& Chaves, V. L. (2004). Prevalência da desnutrição hospitalar em pacientes internados em um hospital filantrópico em Salvador (BA), Brasil. Revista de Ciências Médicas e Biológicas, 3(2), 194-200. http://dx.doi.org/10.9771/cmbio.v3i2.4425

Riquelme, R., Riquelme, M., Rioseco, M. L., Gómez, V., Cárdenas, G. \& Torres, C. (2008). Neumonía adquirida en la comunidad en el anciano hospitalizado: Aspectos clínicos y nutricionales. Revista Médica de Chile, 136(5), 587-593. https://www.scielo.cl/scielo.php?script=sci_arttext\&pid=S003498872008000500006

Sanchez-Munoz, L. A., Calvo-Reyes, M. C., Majo-Carbajo, Y., Barbado-Ajo, J., De La Fuente, M. M. A., Artero-Ruiz, E. C., Municio-Saldaña \& JimenoCarruez, A. (2010). Mini nutritional assessment (MNA) as nutrition screening tool in internal medicine. Advantages and disadvantages. Revista Clinica Espanola, 210(9), 429-437. 
Research, Society and Development, v. 10, n. 7, e24510716468, 2021

(CC BY 4.0) | ISSN 2525-3409 | DOI: http://dx.doi.org/10.33448/rsd-v10i7.16468

Stratton, R. J., Stroud, M. A., Jackson, A. A. \& Elia, M. (2006). 'Malnutrition Universal Screening Tool'predicts mortality and length of hospital stay in acutely ill elderly. British Journal of Nutrition, 95(2), 325-330. 10.1079/bjn20051622

Thomas, J., Isenring, E. \& Kellett. (2007). Nutritional status and length of stay in patients admitted to an Acute Assessment Unit. Journal of Human Nutrition and Dietetics, 20(4), 320-328. 10.1111/ j.1365-277X.2007.00765.x. 